\title{
Theoretical Study of Projectile Fragmentations in Relativistic Heavy-Ion Re- actions
}

\author{
R. Ogul ${ }^{1}$, H. Imal ${ }^{1}$, A. Ergun ${ }^{1}$, N. Buyukcizmeci ${ }^{1}$, A. S. Botvina ${ }^{2,3}$ and W. Trautmann ${ }^{4}$ \\ ${ }^{1}$ Department of Physics Selcuk University, 42079 Kampus, Konya, Turkey \\ ${ }^{2}$ Institute for Nuclear Research, Russian Academy of Sciences, RU-117312 Moscow, Russia \\ ${ }^{3}$ Frankfurt Institute for Advanced Studies, J. W. Goethe University, D-60438 Frankfurt am Main, Germany, GSI \\ ${ }^{4}$ Helmholtzzentrum für Schwerionenforschung $\mathrm{GmbH}, \mathrm{D}-64291$ Darmstadt, Germany
}

\begin{abstract}
We have investigated and interpreted the production cross sections and isotopic distributions of projectile-like residues in the reactions ${ }^{124} \mathrm{Sn}+{ }^{124} \mathrm{Sn}$ and ${ }^{112} \mathrm{Sn}+{ }^{112} \mathrm{Sn}$ at an incident beam energy of 1 $\mathrm{GeV} /$ nucleon measured with the FRS fragment separator at the GSI laboratory. For the interpretation of the data, calculations within the statistical multifragmentation model (SMM) for an ensemble of excited sources were performed with ensemble parameters. The possible modification of symmetry energy parameter, in the multifragmentation region at the low density and hot freeze-out environment, is studied. It is reconfirmed that a significant reduction of the symmetry energy term is found necessary to reproduce experimental results at these conditions. We have also found a decreasing trend of the symmetry energy for large neutron-rich fragments of low excitation energy which is interpreted as a nuclear-structure effect.
\end{abstract}

\section{Introduction}

Nuclear multifragmentation has similarity to the collapse and explosions of massive stars. By this reason, the importance of isotopic effects in nuclear multifragmentation are related to astrophysical processes such as supernova simulations and neutron star models. The midperipheral heavyion collisions at relativistic energies provide us the possibility to study the production of isotopes as a result fragmentation and multifragmentation of excited nuclei within statistical multifragmentation model (SMM)[1]. Recently the experiments for two symmetric systems ${ }^{124} \mathrm{Sn}+{ }^{124} \mathrm{Sn}$ and ${ }^{112} \mathrm{Sn}+{ }^{112} \mathrm{Sn}$ were performed at GSI by FRS collaboration, both at an incident beam energy of $1 \mathrm{~A} \mathrm{GeV}$. The high-resolution magnetic FRagment Separator (FRS) spectrometer was used for separation and extraction of the reaction products [2]. The initial neutron to proton ratios $(\mathrm{N} / \mathrm{Z})$ of the symmetric systems are 1.24 for ${ }^{112} \mathrm{Sn}$ and, 1.48 for ${ }^{124} \mathrm{Sn}$. The isotopic cross sections of the measured fragments for these two reactions were tabulated in Ref.[2]. Previously, we have already analyzed similar reactions: In particular, the ALADIN data were successfully interpreted [3]. On the other hand we have demonstrated in our previous works $[4,5]$, that in order to reproduce the $N / Z$ values, the isoscaling parameters and isotope content of fragments observed in the ALADIN and MSU experimental data, one needs a considerable reduction of the fragment symmetry energy. In this short presentation, we show the results of the analysis of the symmetry energy with the new data on the projectile fragmentation at $1 \mathrm{GeV} /$ nucleon. In the present work, we demon- strate that the same approach can be used to describethe recent FRS data for similar projectiles and that comparable results concerning the required model parameters are obtained. With the new FRS data, the analysis can be extended to include the distributions of heavier isotopes up to close to the initial projectile mass. As shown previously, the symmetry energy represents the main model parameter governing the mean $N / Z$ values, the isoscaling parameters, and the isotopic composition of the fragment yields.

\section{Charge and Isotopic Distribution}

The correlations between the production cross section and charge of the final fragments are shown in Fig. 1 together with the experimental results, which we extract by summing up isotope yields given in the tables of Ref. [2]. For the quantitative comparison of the present results with experimental data, the SMM ensemble calculations were globally normalized with respect to the measured $\mathrm{Z}$ cross sections in the interval $20 \leq \mathrm{Z} \leq 25$ for which the agreement is best. The obtained factors are $0.00334 \mathrm{mb}$ and $0.00344 \mathrm{mb}$ per theoretical event for ${ }^{124} \mathrm{Sn}$ and ${ }^{112} \mathrm{Sn}$ projectiles, respectively. One can see a considerable difference between the experimental data and the theory. In this respect we must take into account that not all isotopes were measured in the experiment. This causes a distortion of the general form of the charge distribution well known since long ago for high energy reactions.

Possible modifications for the symmetry energy term can be investigated by means of the isotopic distributions of projectile fragments, as well. For example, we show in 


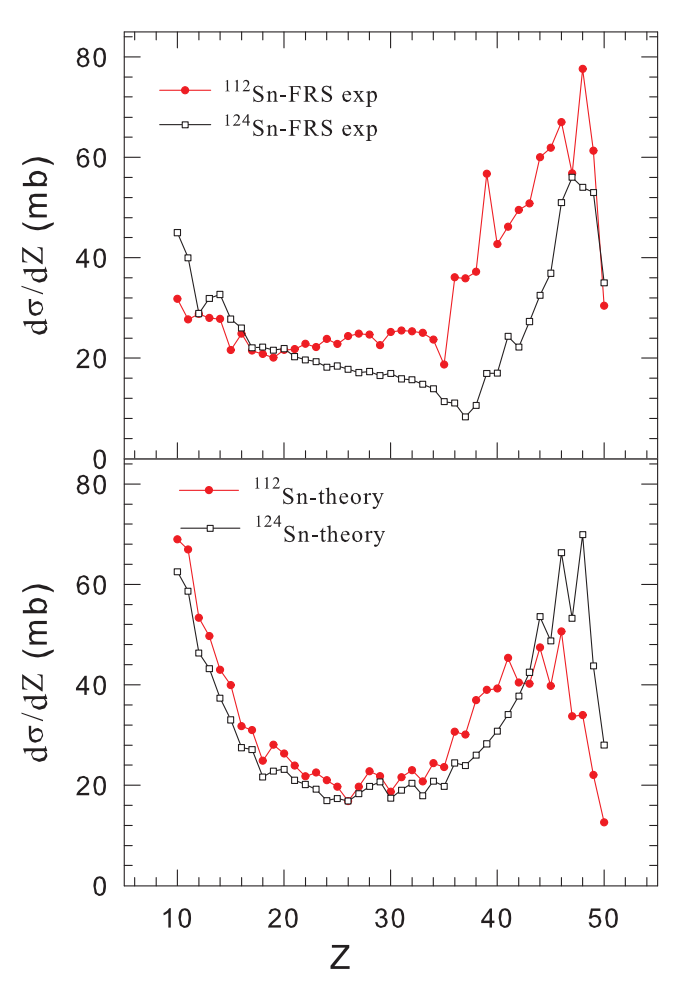

Figure 1. Top panel: production cross sections of the measured projectile fragments as a function of the fragment's $Z$ (from Ref.1). Bottom panel: as obtained from ensemble calculations with standard parameters.

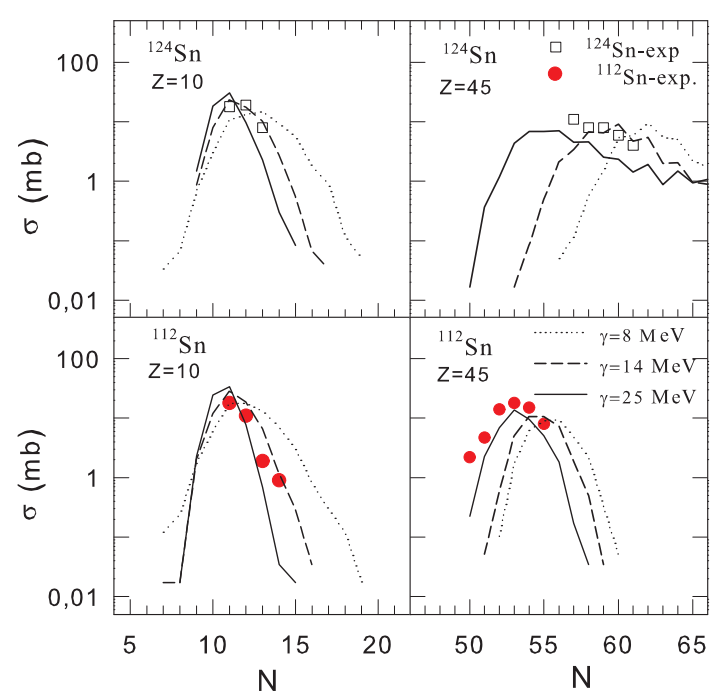

Figure 2. Predicted (lines) and measured (symbols), isotope distributions for final fragments of the projectiles ${ }^{124} \mathrm{Sn}$ and ${ }^{114} \mathrm{Sn}$ with atomic numbers $Z=10$ and $Z=45$, for various symmetry energy values.
Fig. 2 the variation of isotopic distribution with symmetry energy for the extreme values as $Z=10$ and $Z=45$. It is seen that the modified calculations for $Z=10$ (small fragments) agree well with the data at $\gamma=14$, while the calculations with $\gamma=25$ better reproduce the data for $Z=45$ (very large fragments). It is also seen that the width of the distributions is influenced by the symmetry term, such that the isotopic distribution widens considerably with decreasing $\gamma$ values. Details of whole calculations are given in Ref.[6].

\section{Conclusions}

It is seen that the neutron-rich side of isotopic curves is much more sensitive to symmetry energy term for both projectiles, and this sensitivity is considerably larger in the case of neutron-rich ${ }^{124} \mathrm{Sn}$. Therefore, we can conclude that the symmetry energy of fragments should be specially important for the nuclear species close to the neutron dripline. It is confirmed that a significant reduction of the symmetry term coefficient is found necessary to reproduce the isotopic distributions. This is in agreement with our previous results obtained by means of isoscaling and N/Z calculations for the interpretation of ALADIN [3] and MSU data $[4,5]$.

This work was supported by TUBITAK with the research project number 113F058. R.O. thanks the Scientific Research Coordination of Selcuk University (BAP) for the conference support.

\section{References}

[1] J.P. Bondorf, A.S. Botvina et al., Phys. Rep. 257, 133 (1995)

[2] V. Fohr, A. Bacquias et al., Phys. Rev. C 84, 054605 (2011)

[3] R. Ogul, A.S. Botvina et al., Phys. Rev. C 83, 024608 (2011)

[4] R. Ogul, et al., J. Phys. G: Nucl. Part. Phys. 36, 115106 (2009)

[5] N. Buyukcizmeci, et al., J. Phys. G: Nucl. Part. Phys. 39, 115102 (2012)

[6] H. Imal, A. Ergun, N. Buyukcizmeci, R. Ogul, A. S. Botvina and W. Trautmann, nucl-th arXiV:1403.4786 